\title{
Synthesis of Polyesters by Direct Polycondensation with Picryl Chloride
}

\author{
Hozumi TanaKa, Yuki Iwanaga, Guo-chuang Wu, \\ Kohei SANUI, and Naoya OgATA \\ Department of Chemistry, Sophia University, \\ 7-1 Kioi-cho, Chiyoda-ku, Tokyo 102, Japan
}

(Received April 3, 1982)

\begin{abstract}
The polycondensation reaction of various dicarboxylic acids and diols such as terephthalic acid and ethylene glycol occurred at room temperature in pyridine in the presence of picryl chloride to give polyesters in high yield. Reaction conditions, basic solvents, temperatures, concentrations of monomer, and amount of picryl chloride were investigated. A combination of picryl chloride and pyridine was found to be satisfactory for the polyester synthesis. The polycondensation reaction possibly proceeds through the formation of an active ester from picrylic acid and carboxylic acid, followed by esterification with hydroxyl group. Yields of polyester from 2,5-pyridinedicarboxylic acid and 1,10-decanediol by this direct polycondensation reached up to $89 \%$, and the solution viscosity of the polyester was approximately 1.0 under optimum reaction conditions. The polyester obtained from terephthalic acid and 1,5-pentanediol had a higher solution viscosity than those from various combinations of dicarboxylic acids and diols. Aliphatic dicarboxylic acid such as adipic acid yielded polyester having a lower solution viscosity than aromatic dicarboxylic acid.
\end{abstract}

KEY WORDS Direct Polycondensation / Polyester / Picryl Chloride / Pyridine / Terephthalic Acid / 2,5-Pyridinedicarboxylic Acid / Diols / Bisphenol A /

Polyester synthesis is generally carried out at elevated temperatures above $200^{\circ} \mathrm{C}$ under high vacuum in order to eliminate volatile products and thus shift the equilibrium towards the formation of polymer. On the other hand, various routes are available for the synthesis of polyamides under mild conditions $^{1-6}$ by which polycondensation can be enhanced using active monomers such as acid chlorides $^{7}$ or activated esters. ${ }^{8}$ Very few reports have been published on the synthesis of polyesters under mild conditions.

It was previously reported ${ }^{9}$ that aromatic polyesters can be obtained by heating a mixture of dicarboxylic acids and aromatic diols at temperatures above $100^{\circ} \mathrm{C}$ in the presence of triphenylphosphine in pyridine. It was recently found ${ }^{10}$ that aromatic polyesters can be obtained in a quantitative yield under mild conditions by reacting $m$ hydroxybenzoic acid in the presence of triphenylphosphine with poly-halo compounds such as hexachloroethane in a pyridine solution. It was reported in the previous paper ${ }^{11}$ that polyamides and polyesters were formed in the presence of picryl chloride under mild conditions. Various polyesters were prepared by the synthesis method shown below, using picryl chloride as a condensation agent.

$$
\begin{aligned}
& \mathrm{HO}-\mathrm{R}-\mathrm{OH}+\mathrm{HOOC}-\mathrm{R}^{\prime}-\mathrm{COOH}+\mathrm{O}_{2}-\mathrm{NO}_{2}^{\mathrm{NO}} \\
& \underset{\text { in Pyridine }}{\longrightarrow}+\mathrm{O}-\mathrm{R}-\mathrm{OCO}-\mathrm{R}^{\prime}-\mathrm{CO}+\mathrm{Py} \cdot \mathrm{HCl}+\mathrm{O}_{2}
\end{aligned}
$$


H. TANAKA et al.

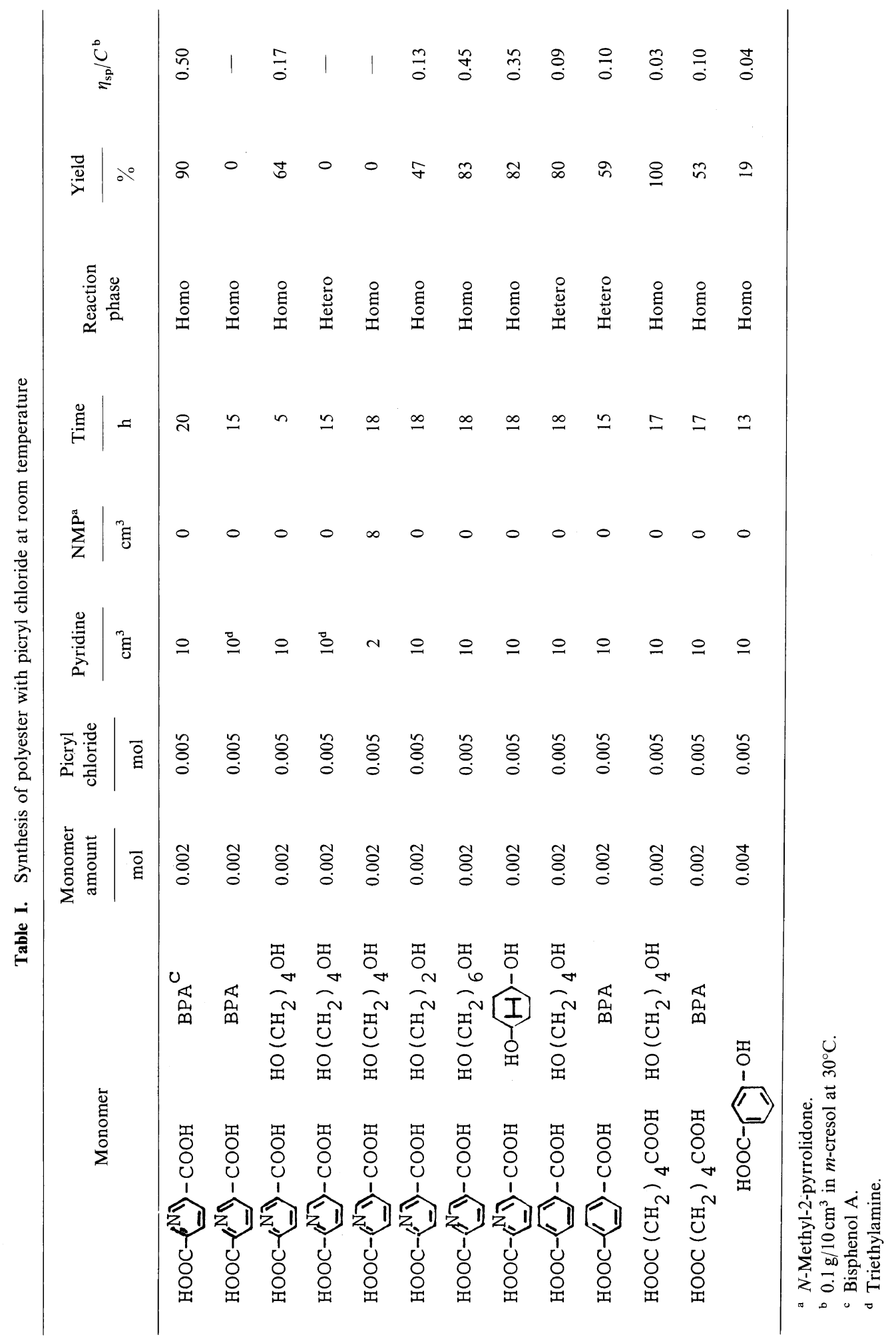


This paper describes the investigation of the optimum reaction conditions for the synthesis of polyesters from various dicarboxylic acids and diols, where the polycondensation of 2,5-pyridinedicarboxylic acid and 1,10-decanediol is emphasized.

\section{EXPERIMENTAL}

Picryl chloride and other aromatic halocompounds of special grade purity were used as purchased. Solvents were purified by conventional procedrues. The general procedure used for the synthesis of polyester from 2,5-pyridinedicarboxylic acid and 1,10-decanediol is as follows:

0.002 mol of 2,5-pyridinedicarboxylic acid and 1,10 -decanediol was dissolved in $10 \mathrm{~cm}^{3}$ of pyridine and then $0.005 \mathrm{~mol}$ of picryl chloride was added to the solution with stirring at room temperature. As soon as picryl chloride was added, an exothermic reaction took place and the solution took on an orange color. The entire reaction proceeded in a homogeneous phase; when terephthalic acid was used, all the reactions proceeded in a heterogeneous phase. After a given period of time, excess acetone was added to remove the solvent, by-products and unreacted monomers from the polymer which was then isolated by filtration. The polyesters from 2,5pyridinedicarboxylic acid and 1,5-pentanediol or 1,9-nonanediol were separated in ether since these polyesters dissolve in acetone.

The polymer was repeatedly washed with water, followed by low boiling point solvents such as acetone, methanol or ether which are poor solvents for the polyesters.

Solution viscosities of the polyesters were determined in $m$-cresol, while those obtained when using terephthalic acid and ethylene glycol were measured in a mixed solvent of phenol and tetrachloroethane $(1 / 1)$ at $30^{\circ} \mathrm{C}$.

\section{RESULTS AND DISCUSSION}

Table I summarizes the results of the synthesis of the polyesters from various dicarboxylic acids and diols by direct polycondensation in the presence of picryl chloride. This table shows that polyesters were obtained in good yield from 2,5-pyridinedicarboxylic acid (2,5-PDC) and bisphenol A or 1,4cyclohexanediol, while the combination of aliphatic

Table II. Direct polycondensation of terephthalic acid or 2,5-pyridine dicarboxylic acid with various diols at room temperature ${ }^{a}$

\begin{tabular}{|c|c|c|c|c|}
\hline \multicolumn{2}{|c|}{ Monomer } & \multirow{2}{*}{$\frac{\text { Time }}{\mathrm{h}}$} & \multicolumn{2}{|c|}{ Polymer } \\
\hline $\begin{array}{c}\mathrm{HOOC}-\mathrm{R}-\mathrm{COOH} \\
\mathrm{R}\end{array}$ & $\underset{n}{\mathrm{HO}}\left(\underset{\mathrm{CH}}{\left.\mathrm{CH}_{2}\right)_{n} \mathrm{OH}}\right.$ & & Yield $/ \%$ & $\eta_{\mathrm{sp}} / C^{\mathrm{b}}$ \\
\hline & 2 & 15 & 33 & 0.05 \\
\hline & 3 & 15 & 85 & 0.12 \\
\hline & 4 & 15 & 84 & 0.19 \\
\hline & 5 & 15 & 86 & 0.19 \\
\hline & 6 & 15 & 87 & 0.43 \\
\hline & 9 & 14 & 57 & 0.33 \\
\hline & 10 & 14 & 95 & 0.22 \\
\hline & 12 & 16 & 77 & 0.22 \\
\hline & 2 & 18 & 47 & 0.13 \\
\hline & 3 & 16 & 73 & 0.33 \\
\hline & 4 & 16 & 75 & 0.53 \\
\hline & 5 & 16 & 79 & 0.45 \\
\hline & 6 & 16 & 78 & 0.56 \\
\hline & 9 & 16 & 36 & 0.17 \\
\hline & 10 & 16 & 89 & 0.72 \\
\hline & 12 & 16 & 77 & 0.63 \\
\hline
\end{tabular}

a Amount of monomer $=0.002 \mathrm{~mol}$; amount of picryl chloride $=0.005 \mathrm{~mol}$; solvent, $10 \mathrm{~cm}^{3}$ of pyridine.

b $0.1 \mathrm{~g} / 10 \mathrm{~cm}^{3}$ in $m$-cresol at $30^{\circ} \mathrm{C}$. 
dicarboxylic acid and diols failed to give satisfactory results in terms of the solution viscosities.

When triethylamine was used in place of pyridine, no polymer was obtained. The polycondensation of $m$-hydroxybenzoic acid resulted in a lower yield than that of 2,5-PDC and ethylene glycol.

Table II shows the results of the polycondensation of aliphatic diols of various methylene units with terephthalic acid or 2,5-PDC at room temperature.

Solution viscosities reached a maximum value of 0.58 when 1,5-pentanediol was used along with terephthalic acid, and those of the polyesters decreased with increasing chain length of the methylene units in diols. On the other hand, 2,5-PDC yielded polyesters with aliphatic diols in good yield. The solution viscosities, which increased almost in proportion to the number of methylene units of diols, obtained a value of 0.72 except when using 1,9-nonanediol. The fluctuation in the solution viscosity of these polyesters may possibly have been caused by solvent affinity of the polyesters in pyridine, thus terminating chain growth by the precipitation of the polyesters out of the reaction phase. Since the polyester obtained when using terephthalic acid and ethylene glycol apparently did not dissolve in pyridine, the solution viscosity was as low as 0.05 .

Figure 1 indicates the rate of the polycondensation of 2,5-PDC with 1,10-decanediol which took place very rapidly in the presence of

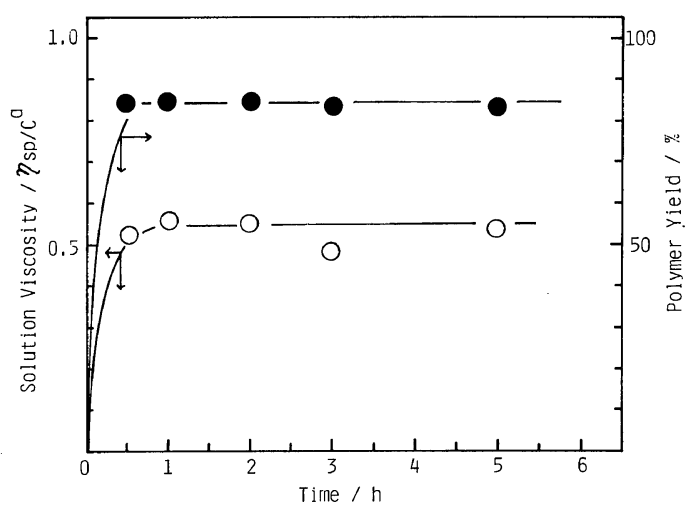

Figure 1. Rate of polycondensation of 2,5-pyridinedicarboxylic acid with 1,10-decanediol at room temperature: Amount of monomer $=0.002 \mathrm{~mol}$; amount of picryl chloride $=0.005 \mathrm{~mol}$; solvent, $10 \mathrm{~cm}^{3}$ of pyridine. ${ }^{\mathrm{a}} 0.1 \mathrm{~g} /$ $10 \mathrm{~cm}^{3}$ in $m$-cresol at $30^{\circ} \mathrm{C}$. picryl chloride in pyridine. The solution viscosity of the polyester reached a constant value within $30 \mathrm{~min}$ and the growing reaction was completed with $1 \mathrm{~h}$ even at room temperature.

Figure 2 indicates the effect of the amount of picryl chloride to the monomer on the polycondensation of 2,5-PDC and 1,10-decanediol. The solution viscosity of the resulting polyesters increased with increasing amounts of picryl chloride, reaching a value of 0.9 and the optimum molar ratio of picryl chloride to the monomer was found to be

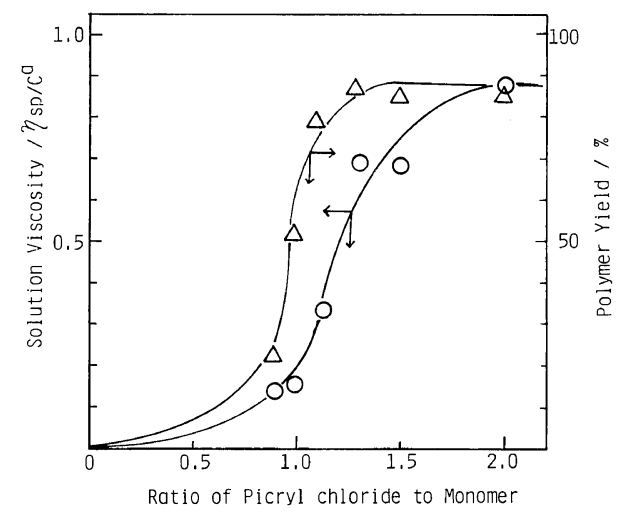

Figure 2. Effect of amount of picryl chloride on polycondensation of 2,5-pyridinedicarboxylic acid with 1,10decanediol at room temperature: Amount of monomer $=0.002 \mathrm{~mol}$; time $=1 \mathrm{~h} .{ }^{\mathrm{a}} 0.1 \mathrm{~g} / 10 \mathrm{~cm}^{3}$ in $\mathrm{m}$ cresol at $30^{\circ} \mathrm{C}$.

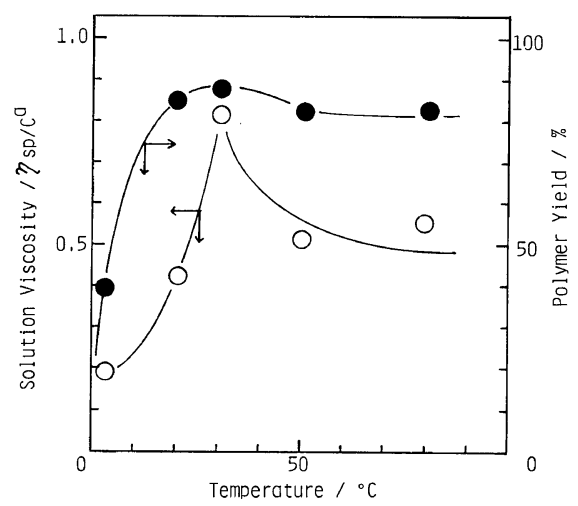

Figure 3. Effect of temperature on polycondensation of 2,5-pyridinedicarboxylic acid with 1,10-decanediol: Amount of monomer $=0.002 \mathrm{~mol}$; amount of picryl chloride $=0.005 \mathrm{~mol}$; solvent, $10 \mathrm{~cm}^{3}$ of pyridine. ${ }^{\mathrm{a}} 0.1 \mathrm{~g} /$ $10 \mathrm{~cm}^{3}$ in $\mathrm{m}$-cresol at $30^{\circ} \mathrm{C}$. 
2.0. When an excess amount of picryl chloride was added, separation of the polyester became difficult because of the formation of the pyridinium salt of picryl chloride with pyridine.

Figure 3 summarizes the dependence of the solution viscosities of the polyesters on the reaction temperatures. The reaction temperature influenced the solution viscosity which showed a maximum at $30^{\circ} \mathrm{C}$, as can be seen in Figure 3. The polycondensation at a temperature of $50^{\circ} \mathrm{C}$ gave the polyesters having lower solution viscosities, presumably owing to thermal degradation of reaction intermediate which might increase at higher temperatures.

Figure 4 shows the effect of the monomer concentration on the solution viscosity of the polyester. The monomer concentration was found to have a significant influence on the solution viscosities of the polyesters. When the polycondensation reaction of 2,5-PDC with 1,10-decanediol was carried out at a concentration of $0.6 \mathrm{~mol} \mathrm{dm}^{-3}$, the resulting polyester had a solution viscosity of $c a$. 1.0 , while at a monomer concentration of more than $0.8 \mathrm{~mol}$ $\mathrm{dm}^{-3}$, the solution viscosity of the resulting polyester decreased with increasing the monomer concentration in pyridine, presumably because the polycondensation reaction proceeded in a heterogeneous phase.

Acid acceptors were also an important factor in obtaining polyesters of high solution viscosity. Various basic solvents were used both as solvents

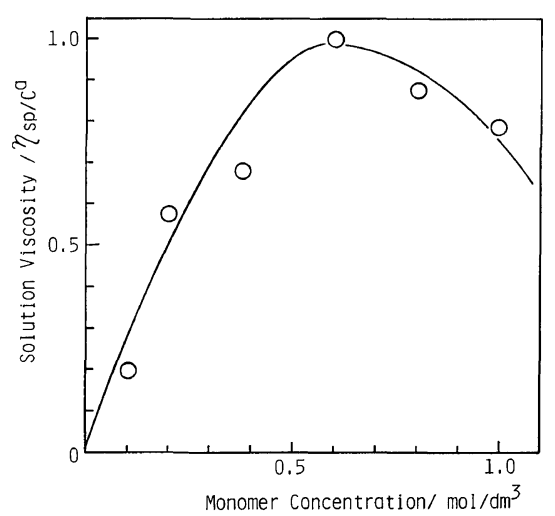

Figure 4. Effect of monomer concentration on polycondensation of 2,5-pyridinedicarboxylic acid with 1,10-decanediol at room temperature: Solvent, pyridine; ratio of picryl chloride to monomer $=1.25$; time $=1 \mathrm{~h}$. ${ }^{\text {a }} 0.1 \mathrm{~g} / 10 \mathrm{~cm}^{3}$ in $m$-cresol at $30^{\circ} \mathrm{C}$. and acid acceptors for the polycondensation of 2,5PDC and 1,10-decanediol at room temperature. As shown in Table III, pyridine was a specific solvent to yield a polyester of high solution viscosity from 2,5-PCD with 1,10-decanediol. The yield and solution viscosity of the polyester may have depended on the formation of a reaction intermediate which was influenced by the basicity and steric hindrance of pyridine derivatives.

Pyridine might be favorable to a better solubility of the polyester, and enhance the growth reaction of the polymer chains.

Table III. Effect of solvent on polycondensation of 2,5-pyridine dicarboxylic acid with 1,10 -decanediol at room temperature ${ }^{\mathrm{a}}$

\begin{tabular}{lrrc}
\hline \multirow{1}{*}{ Solvents } & $\mathrm{p} K_{\mathrm{a}}$ & \multicolumn{2}{c}{ Yield } \\
\cline { 3 - 3 } & & \multirow{2}{*}{$\eta_{\mathrm{sp}} / C^{\mathbf{b}}$} \\
& & & \\
\hline 2-Chloropyridine & 0.49 & 41 & - \\
Quinoline & 4.81 & 87 & 0.06 \\
Pyridine & 5.25 & 89 & 0.69 \\
3-Picoline & 5.63 & 82 & 0.43 \\
2-Picoline & 5.94 & 0 & - \\
4-Picoline & 6.03 & 0 & - \\
2,6-Lutidine & 6.60 & 33 & 0.12 \\
Triethylamine & 10.75 & 0 & - \\
\hline
\end{tabular}

a Amount of monomer $=0.002 \mathrm{~mol}$; amount of picryl chloride $=0.005 \mathrm{~mol}$; time $=1 \mathrm{~h}$.

b $0.1 \mathrm{~g} / 10 \mathrm{~cm}^{3}$ in $m$-cresol at $30^{\circ} \mathrm{C}$.

Table IV. Direct polycondensation of 2,5-pyridinedicarboxylic acid with 1,10-decanediol with various aromatic halo-compounds at room temperature ${ }^{\text {a }}$

\begin{tabular}{|c|c|c|c|}
\hline ArX & $\frac{\text { Time }}{\mathrm{h}}$ & $\frac{\text { Yield }}{\%}$ & $\eta_{\mathrm{sp}} / C^{\mathrm{b}}$ \\
\hline & 15 & 39 & 0.11 \\
\hline $\mathrm{O}_{2} \mathrm{~N}$ & 15 & 0 & - \\
\hline & 18 & 89 & 0.72 \\
\hline
\end{tabular}

a Amount of monomer $=0.002 \mathrm{~mol}$; amount of $\mathrm{ArX}=$ $0.005 \mathrm{~mol}$.

b $0.1 \mathrm{~g} / 10 \mathrm{~cm}^{3}$ in $m$-cresol at $30^{\circ} \mathrm{C}$. 
Results of the polycondensation of 2,5-PDC with 1,10 -decanediol in the presence of various aromatic nitro-halogen compounds as condensation agents are summarized in Table IV.

No polymer was obtained by 2,4-dinitrochlorobenzene, while 2,4-dinitrofluorobenzene yielded a polyester having a solution viscosity of 0.11 in pyridine. Therefore, picryl chloride was found to be a good initiator for direct polycondensation in the synthesis of polyesters from 2,5-PDC and 1,10decanediol. A previous paper ${ }^{12}$ proposed the reaction mechanism of direct polycondensation of 4aminobenzoic acid by picryl chloride in pyridine. The same mechanism could be applied to the polyester synthesis.

The active intermediate is presumed to be trinitrophenyl ester as shown below:

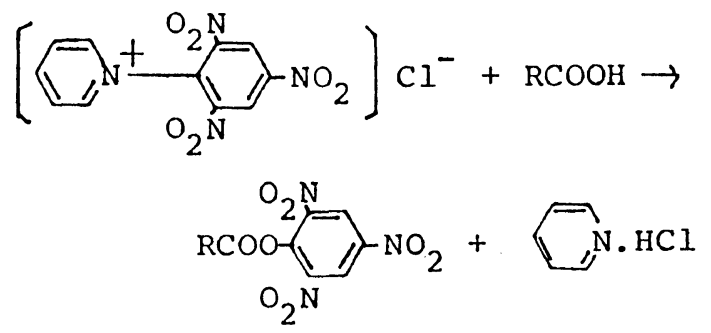

This active ester reacts with alchol to form a new ester linkage through an ester exchange reaction.<smiles>[R]OCC[PH2+][O+]c1cc([N+](=O)[O-])cc([N+](=O)[O-])c1</smiles>

The active intermediate, trinitrophenyl ester may react with the unreacted acid to form acid anhydride, which may cause the reaction to terminate and prevent the growth of polymer chains, owing to the loss of stoichiometry of the functional groups.

Results of the order of reagent addition in the polycondensation reaction of 2,5-pyridinedicarboxylic acid with 1,10-decanediol in pyridine are shown in Table $\mathrm{V}$.

A polyester of a higher solution viscosity (0.69) was obtained when the reagents were added all at
Table V. Effect of addition of reagents on the polycondensation reaction

\begin{tabular}{ccc}
\hline & Yield & \\
\cline { 2 - 2 } Addition order & $\%$ & $\eta_{\text {sp }} / C^{\mathrm{e}}$ \\
& $\%$ & \\
\hline$\left(\mathrm{Pi}^{\mathrm{a}}+\mathrm{PD}^{\mathrm{b}}+\mathrm{DD}^{\mathrm{c}}+\mathrm{Py}^{\mathrm{d}}\right)$ & 89 & 0.69 \\
$(\mathrm{Pi}+\mathrm{Py})^{1}+(\mathrm{PD}+\mathrm{DD})^{2}$ & 85 & 0.38 \\
$(\mathrm{Pi}+\mathrm{Py})^{1}+\mathrm{PD}^{2}+\mathrm{DD}^{3}$ & 0 & - \\
$(\mathrm{Pi}+\mathrm{PD}+\mathrm{Py})^{1}+\mathrm{DD}^{2}$ & 0 & - \\
\hline
\end{tabular}

a Picryl chloride.

b 2,5-Pyridinedicarboxylic acid.

c 1,10-Decanediol.

d Pyridine.

e $0.1 \mathrm{~g} / 10 \mathrm{~cm}^{3}$ in $m$-cresol at $30^{\circ} \mathrm{C}$.

Addition order: The following reagents were added to the reaction mixture after $1 \mathrm{~h}$.

once compared to when they were added one by one.

In conclusion, it was found that conditions were optimum at a monomer concentration of $0.003 \mathrm{~mol}$ for both dicarboxylic acid and diol in the presence of $0.009 \mathrm{~mol}$ of picryl chloride in $10 \mathrm{~cm}^{3}$ of pyridine. A polyester having a solution visocisty of $c a .1 .0$ was obtained at $30^{\circ} \mathrm{C}$ within $5 \mathrm{~h}$.

\section{REFERENCES}

1. N. Ogata and H. Tanaka, Polym. J., 2, 672 (1971).

2. N. Ogata and H. Tanaka, Polym. J., 3, 365 (1973).

3. N. Ogata and H. Tanaka, Polym. J., 6, 461 (1974).

4. N. Ogata, K. Sanui, and M. Harada, J. Polym. Sci., Polym. Chem. Ed., 17, 2401 (1979).

5. N. Yamazaki, F. Higashi and J. Kawabata, J. Polym. Sci., Polym. Chem. Ed., 12, 2149 (1974).

6. G. Wu, H. Tanaka, K. Sanui, and N. Ogata, J. Polym. Sci., Polym. Lett. Ed., 19, 343 (1981).

7. P. W. Morgan, "Condensation Polymers by Interfacial and Solution Methods," Interscience, New York, 1965.

8. K. Sanui, S. Tanaka, and N. Ogata, J. Polym. Sci., Polym. Chem. Ed., 15, 1107 (1977).

9. F. Higashi, K. Kubota, and M. Sekizuki, Polym. Prepr., Jpn., 29, 65 (1980).

10. N. Ogata, K. Sanui, H. Tanaka, and S. Yasuda, Polym. J., 13, 989 (1981).

11. H. Tanaka, G. Wu, Y. Iwanaga, K. Sanui, and N. Ogata, Polym. J., 14, 331 (1982).

12. H. Tanaka, G. Wu, Y. Iwanaga, K. Sanui, and N. Ogata, Polym. J., 14, 635 (1982). 